\title{
UNVEILING THE NATURE OF SUBMILLIMETER GALAXY SXDF 850.6
}

\author{
B. Hatsukade ${ }^{1}$, D. Iono ${ }^{2}$, T. Akiyama ${ }^{3}$, M. Yoshikawa ${ }^{3}$, J. S. Dunlop ${ }^{4}$, R. J. Ivison ${ }^{4,5}$, A. B. Peck ${ }^{6}$, S. Ikarashi ${ }^{1}$, A. Biggs ${ }^{7}$, \\ H. Ezawa ${ }^{8}$, H. Hanami ${ }^{9}$, P. Ho $^{10}$, D. H. Hughes ${ }^{11}$, R. Kawabe ${ }^{2}$, K. Kohno ${ }^{1,12}$, S. Matsushita ${ }^{10}$, K. NaKanishi $^{2}$, \\ N. Padilla ${ }^{13}$, G. PetitPas ${ }^{14}$, Y. TAmura ${ }^{2}$, J. WagG ${ }^{11,15,16}$, D. J. Wilner ${ }^{16}$, G. W. Wilson ${ }^{17}$, T. YAMAdA $^{3}$, And M. S. Yun ${ }^{17}$

\section{INTRODUCTION}

Millimeter/submillimeter surveys have revolutionized observational cosmology by uncovering a substantial new population of millimeter/submillimeter-bright dusty starburst galaxies at high redshifts (SMGs; e.g., Smail et al. 1997; Barger et al. 1998; Hughes et al. 1998; Scott et al. 2002, 2008; Coppin et al. 2006; Greve et al. 2004; Laurent et al. 2005; Perera et al. 2008; Austermann et al. 2009; Tamura et al. 2009; see also Blain et al. 2002 for a review). The energy source of millimeter/submillimeter emission is primarily from intense star formation activity, with star formation rates (SFRs) of $100 M_{\odot} \mathrm{yr}^{-1}$ to several 1000 $M_{\odot} \mathrm{yr}^{-1}$, and possibly partially from an active galactic nucleus (AGN; e.g., Alexander et al. 2005). While spectroscopic observations of radio-identified SMGs find a median redshift of 2.2 (Chapman et al. 2005), several SMGs have now been found up to and beyond $z=4$ (e.g., Capak et al. 2008; Coppin et al. 2009; Daddi et al. 2009). There is a hypothesis that SMGs are progenitors of present-day massive ellipticals (e.g., Lilly et al. 1999; Smail et al. 2004); however, little is known about their evolution process.

While multi-wavelength analysis is essential in order to understand the nature of SMGs, the coarse angular resolution of single-dish telescopes prevents a precise determination of the exact optical/NIR counterparts. One of the most successful ways to pinpoint the location of the submillimeter emission is to obtain high-resolution, deep radio imaging (e.g., Ivison et al. 1998, 2000, 2002; Smail et al. 2000; Barger et al. 2000). Although this technique reveals robust radio counterparts of $\sim 50 \%-80 \%$ of submillimeter sources (e.g., Ivison et al. 2005, 2007; Wagg et al. 2009), sometimes multiple radio counterpart candidates are found for a source.

The most accurate means of achieving high-precision astrometry on the submillimeter emission is clearly to observe with high angular resolution at the wavelength of the original detection. In this respect, the Submillimeter Array (SMA; Ho et al. 2004) has proved to be a powerful instrument (Iono et al. 2006a, 
2006b; Wang et al. 2007; Younger et al. 2007, 2008, 2009; Ivison et al. 2008).

Here, we present the results from the SMA observations toward an $8 \mathrm{mJy}$ submillimeter source, SXDF 850.6, detected in the SCUBA Half Degree Extragalactic Survey (SHADES). SHADES has observed a large area of the sky $\left(720 \mathrm{arcmin}^{2}\right)$ with high sensitivity $(1 \sigma \sim 2 \mathrm{mJy})$ with the purpose of obtaining a statistically significant unbiased sample of submillimeter sources (Mortier et al. 2005; Coppin et al. 2006). The regions covered by SHADES are divided between two fields, the Lockman Hole and the Subaru/XMM-Newton Deep Field. SXDF 850.6 is a source in the SXDF with multiple optical, IR, and radio counterparts (Ivison et al. 2007; Clements et al. 2008), but no established submillimeter source identification. The strongest radio emission has no confirmed optical counterpart, but the two secondary radio peaks both have apparent optical associations.

Section 2 outlines the observational and calibration details, and the results are presented in Section 3. In Section 4, multiwavelength data are described. The results of SED model fitting using the photometry are presented in Section 5. In Section 6, we discuss the nature of SXDF 850.6. A summary is presented in Section 7. Throughout the paper, magnitudes are in the $A B$ system, and we adopt a cosmology with $H_{0}=70 \mathrm{~km} \mathrm{~s}^{-1} \mathrm{Mpc}^{-1}$, $\Omega_{\mathrm{M}}=0.3$, and $\Omega_{\Lambda}=0.7$.

\section{OBSERVATIONS AND DATA REDUCTION}

SXDF 850.6 was observed on 2004 September 21 and October 7, and on 2005 October 9 and 14, using a compact configuration with 7-8 antennas of the SMA. The phase center was positioned at the submillimeter source centroid, which is at $\alpha(\mathrm{J} 2000)=02^{\mathrm{h}} 17^{\mathrm{m}} 29^{\mathrm{s}} .80$ and $\delta(\mathrm{J} 2000)=-05^{\circ} 03^{\prime} 26^{\prime \prime} 00$. The unprojected baseline length ranged from $23 \mathrm{~m}$ to $139 \mathrm{~m}$. The SMA correlator was equipped with $2 \mathrm{GHz}$ total bandwidth in each sideband, yielding a total of $4 \mathrm{GHz}$ bandwidth for continuum observations. A continuum channel was generated by vector averaging all of the channels after calibration. The SIS receivers were tuned to $345 \mathrm{GHz}$ for the upper sideband, yielding $335 \mathrm{GHz}$ for the lower sideband. Interferometric pointing was checked at the beginning of the track and the pointing offsets were usually within $\sim \pm 5^{\prime \prime}$ (15\% of the primary beam) for all antennas. We used an integration time of $30 \mathrm{~s}$.

The raw SMA data were calibrated using the MIR package (Scoville et al. 1993). Passband calibration was done using bright QSOs and planets observed during the track. Antennabased phase calibration was done using J0238+166 (1.02 Jy), J0423-013 (1.67 Jy), and J0132-169 (0.84 Jy). The flux levels of all sources were normalized using the quasar flux estimates derived from the primary flux standard Uranus. Imaging was carried out in MIRIAD (Sault et al. 1995). Maximum sensitivity was achieved by adopting natural weighting, which gave a final synthesized beam size of $2^{\prime \prime} .32 \times 2^{\prime \prime} .19($ P.A. $=79.1)$ and an rms noise of $1.2 \mathrm{mJy}$. Because the source is close to zero in declination, the $u v$ coverage is undersampled in the northsouth direction and the resultant synthesized beam has multiple sidelobes at the $30 \%$ level along the north-south direction about $14^{\prime \prime}$ away from the synthesized beam. The final map was made after adding the data from all four days and two sidebands, and corrected for the attenuation by the $35^{\prime \prime}$ primary beam of the SMA. The astrometric accuracy is likely dominated by the low signal-to-noise ratio $(\mathrm{S} / \mathrm{N})$ of the image, and we assess $\sim 0.4$.

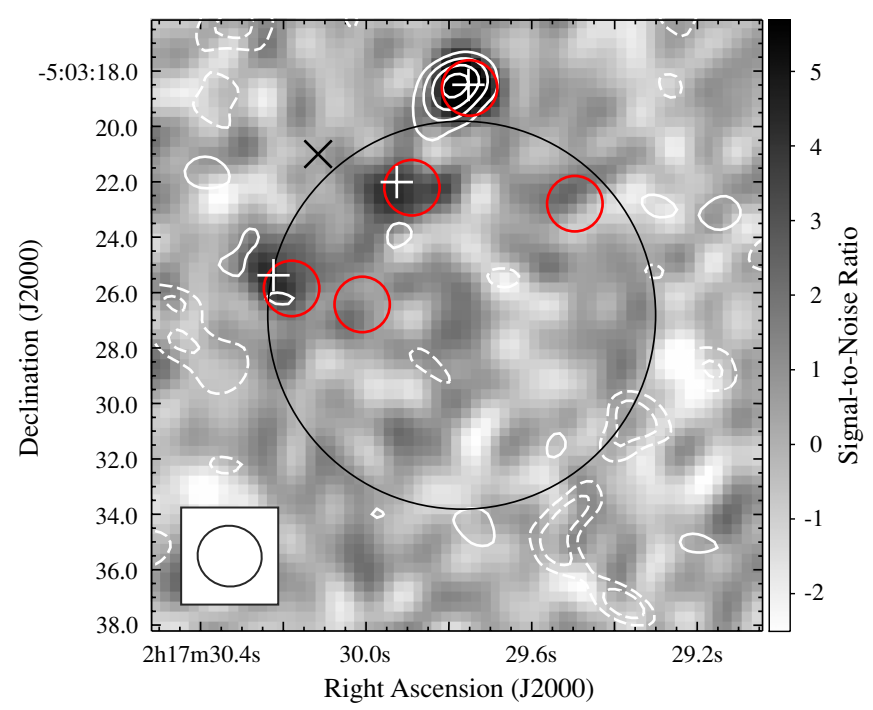

Figure 1. SMA contours overlaid on the VLA $21 \mathrm{~cm}$ image. The contours represent $-3 \sigma,-2 \sigma, 2 \sigma, 3 \sigma, 4 \sigma$, and $5 \sigma$ (where $1 \sigma=1.2 \mathrm{mJy}$ ). The synthesized beam $\left(2{ }^{\prime \prime} .32 \times 22^{\prime \prime} 19\right)$ is shown in lower left. The dark circle represents the approximate size of the SCUBA/JCMT beam (FWHM 14"). The dark cross, white crosses, and red circles are the AzTEC position (S. Ikarashi et al. 2010, in preparation), three radio counterpart candidates in Ivison et al. (2007), and MIPS $24 \mu \mathrm{m}$ sources in the SWIRE catalog, respectively.

\section{RESULTS}

The synthesized map is shown in Figure 1. A source was detected at about $6 \sigma$ significance 8 ". 2 north of the SCUBA coordinates. The source appears slightly elongated in the northeastsouthwest direction but this is likely caused by the low $\mathrm{S} / \mathrm{N}$, and the source is entirely unresolved with the SMA beam. Based on a point source fit to the visibilities, the derived flux is $6.9 \pm 1.2 \mathrm{mJy}$, and the coordinates are $\alpha(\mathrm{J} 2000)=02^{\mathrm{h}} 17^{\mathrm{m}} 29.79$ and $\delta(\mathrm{J} 2000)=-05^{\circ} 03^{\prime} 18^{\prime \prime} .65$. The derived flux is consistent with the SCUBA $850 \mu \mathrm{m}$ flux of $8.15 \pm 2.2 \mathrm{mJy}$ (Coppin et al. 2006) within the uncertainties of both measurements.

\section{MULTI-WAVELENGTH DATA}

Multi-wavelength images around SXDF 850.6 are shown in Figure 2. While an obvious optical counterpart is not seen in the images from $u$ to $z^{\prime}$ bands, the NIR images represented in $J H K$ and longer wavelength images show the presence of a counterpart. The photometry of the counterpart and upper limits at the SMA position are presented in Table 1.

\subsection{Radio and Millimeter}

Ivison et al. (2007) identify three radio counterpart candidates for SXDF 850.6 in their deep VLA $21 \mathrm{~cm}$ map based on the probability analysis method of Downes et al. (1986). All three radio sources have corresponding MIPS $24 \mu \mathrm{m}$ sources (Clements et al. 2008), as shown in Figure 1. The SMA observation reveals that the correct radio counterpart is the most distant source from the SCUBA centroid and the brightest in $24 \mu \mathrm{m}$ emission. This demonstrates the effectiveness of the SMA in identifying SMG counterparts, especially in situations with multiple counterpart candidates.

The radio coordinates derived from reanalysis of the VLA image (V. Arumugam et al. 2010, in preparation) are $\alpha(\mathrm{J} 2000)=$ $02^{\mathrm{h}} 17^{\mathrm{m}} 29^{\mathrm{s}} .755( \pm 0.005)$ and $\delta(\mathrm{J} 2000)=-05^{\circ} 03^{\prime} 18^{\prime \prime} .40\left( \pm 0^{\prime \prime} .08\right)$, 

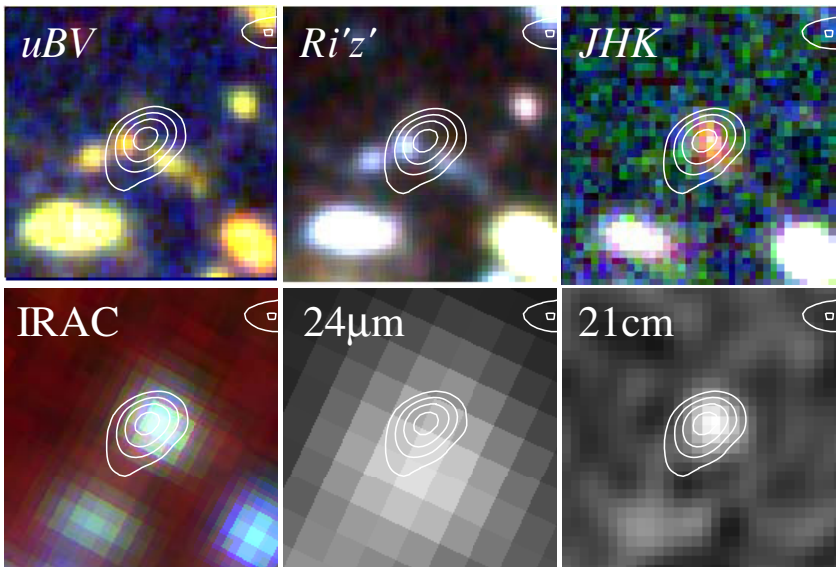

Figure 2. Multi-wavelength images of SXDF 850.6 with the SMA contours $(2 \sigma, 3 \sigma, 4 \sigma$, and $5 \sigma)$. The size of each image is $10^{\prime \prime} \times 10^{\prime \prime}$ and north is up. From left to right: $\mathrm{rgb}$ image of MOSAIC II $/ u$, SuprimeCam $/ B$, and $V ; \operatorname{rgb}$ image of SuprimeCam $/ R, i^{\prime}$, and $z^{\prime} ;$ rgb image of WFCAM $/ J, H$, and $K$; rgb image of IRAC/ch1 (blue), ch2 (green), ch3, and 4 (red); MIPS $24 \mu \mathrm{m}$; VLA $21 \mathrm{~cm}$.

Table 1

Fluxes of SXDF 850.6

\begin{tabular}{lccc}
\hline \hline Band & Flux & Instrument & Ref. \\
\hline$u$ & $<0.0148 \mu \mathrm{Jy}$ & MOSAIC II & 1 \\
$B$ & $<0.0248 \mu \mathrm{Jy}$ & SuprimeCam & 1 \\
$V$ & $<0.0315 \mu \mathrm{Jy}$ & SuprimeCam & 1 \\
$R$ & $<0.0397 \mu \mathrm{Jy}$ & SuprimeCam & 1 \\
$i^{\prime}$ & $<0.0389 \mu \mathrm{Jy}$ & SuprimeCam & 1 \\
$z^{\prime}$ & $<0.0996 \mu \mathrm{Jy}$ & SuprimeCam & 1 \\
$J$ & $1.40_{-0.20}^{+0.23} \mu \mathrm{Jy}$ & WFCAM & 1 \\
$H$ & $3.21_{-0.22}^{+0.23} \mu \mathrm{Jy}$ & WFCAM & 1 \\
$K$ & $9.17 \pm 0.23 \mu \mathrm{Jy}$ & WFCAM & 1 \\
$3.6 \mu \mathrm{m}$ & $29.0 \pm 0.8 \mu \mathrm{Jy}$ & IRAC & 1 \\
$4.5 \mu \mathrm{m}$ & $39.3 \pm 1.1 \mu \mathrm{Jy}$ & IRAC & 1 \\
$5.8 \mu \mathrm{m}$ & $54.3 \pm 1.9 \mu \mathrm{Jy}$ & IRAC & 1 \\
$8.0 \mu \mathrm{m}$ & $38.2 \pm 1.4 \mu \mathrm{Jy}$ & IRAC & 1 \\
$24 \mu \mathrm{m}$ & $630 \pm 30 \mu \mathrm{Jy}$ & MIPS & 1 \\
$70 \mu \mathrm{m}$ & $<30 \mathrm{mJy}^{\prime}$ & MIPS & 2 \\
$160 \mu \mathrm{m}$ & $<200 \mathrm{mJy}^{\prime}$ & MIPS & 2 \\
$450 \mu \mathrm{m}$ & $<81 \mathrm{mJy}^{2}$ & SCUBA & 3 \\
$850 \mu \mathrm{m}$ & $8.15 \pm 2.2 \mathrm{mJy}$ & SCUBA & 3 \\
$880 \mu \mathrm{m}$ & $6.9 \pm 1.2 \mathrm{mJy}$ & SMA & 1 \\
$1100 \mu \mathrm{m}$ & $3.9 \pm 0.5 \mathrm{mJy}$ & AzTEC & 4 \\
$21 \mathrm{~cm}$ & $100.0 \pm 10.6 \mu \mathrm{Jy}$ & VLA & 5 \\
$0.2-12 \mathrm{keV}$ & $<6.3 \times 10^{-15} \mathrm{erg} \mathrm{cm}^{-2} \mathrm{~s}^{-1}$ & $X M M-N e w t o n$ & 6 \\
\hline
\end{tabular}

Note. Limits are $3 \sigma$

References. (1) This work; (2) Flux limits in the SWIRE catalog (J. Surace et al. 2010, in preparation); (3) Coppin et al. 2006; (4) S. Ikarashi et al. 2010, in preparation; (5) V. Arumugam et al. 2010, in preparation (6) FLIX: upper limit server for XMM-Newton data provided by the XMM-Newton Survey Science Centre.

with peak and integrated flux of $99.8 \mu \mathrm{Jy}$ and $100.0 \pm 10.6 \mu \mathrm{Jy}$, respectively.

The close similarity of the peak and integrated flux suggests that the radio emission is unresolved with the $1^{\prime \prime} .79 \times 1$ 1".51 beam $($ P.A. $=6.1)$.

SXDF 850.6 is detected at $1100 \mu \mathrm{m}$ with deboosted flux of $3.9 \pm 0.5 \mathrm{mJy}$ (S. Ikarashi et al. 2010, in preparation) using the AzTEC camera (Wilson et al. 2008) on the Atacama Submillimeter Telescope Experiment (ASTE; Ezawa et al. 2004, 2008). The AzTEC coordinates are consistent with the SMA position within a $2 \sigma$ error circle.

\subsection{Mid-IR}

Spitzer/IRAC and MIPS data are taken from the SpUDS archive. The $3 \sigma$ detection limits are $0.58,0.89,5.7$, and $5.3 \mu \mathrm{Jy}$ in IRAC bands $(3.6,4.5,5.8$, and $8.0 \mu \mathrm{m})$, and $36 \mu \mathrm{Jy}$ in MIPS $24 \mu \mathrm{m}$ band. The photometry was performed using the SExtractor package (Bertin \& Arnouts 1996). The photometry in IRAC bands is conducted with 3 "'.8 diameter aperture, and the photometric zero points of the images are determined using colors of early-type stars between $K$ band and IRAC bands following Lacy et al. (2005). In order to derive total flux, the aperture photometry are corrected by assuming IRAC point-spread functions by a factor of $1.36,1.40,1.65$, and 1.84 , respectively. The photometry in the $24 \mu \mathrm{m}$ band is conducted using $12^{\prime \prime}$ diameter aperture. The aperture magnitude is corrected by a factor of 1.70 .

We find emission at the SMA position in all IRAC bands and MIPS $24 \mu \mathrm{m}$ band. Note that the $24 \mu \mathrm{m}$ flux in Table 1 should be used as an upper limit since sources around the SMA position are blended in the $24 \mu \mathrm{m}$ image (Figure 2).

\section{3. $J H K$}

$J, H$, and $K$ band images are obtained from UKIDSS (UKIRT InfraRed Deep Sky Surveys) Ultra Deep Survey Third Data Release (Laurent et al. 2005). The $3 \sigma$ detection limits are 24.28, 24.08 , and $24.18 \mathrm{mag}$, respectively.

A faint source is detected at the SMA position in all bands. We perform photometry using the SExtractor. Aperture photometry with 1".8 diameter aperture in the $J, H$, and $K$ bands are corrected by difference between the aperture and the total (MAG_AUTO) magnitudes in the $K$ band.

\section{4. $u B V R i^{\prime} z^{\prime}$}

We present a $u$-band image of MOSAIC II on Cerro Tololo Inter-American Observatory $4 \mathrm{~m}$ telescope (N. Fujishiro et al. 2010 , in preparation), $B, V, R, i^{\prime}$, and $z^{\prime}$ images of SuprimeCam on Subaru telescope in Figure 2. $B, V, R, i^{\prime}$, and $z^{\prime}$ band images are obtained from Subaru/XMM-Newton Deep Survey database (SXDS; Furusawa et al. 2008). No galaxy is detected at the SMA position in these bands. The $3 \sigma$ detection limits are 26.00 , $27.94,27.68,27.43,27.45$, and 26.43 mag, respectively.

Near the SMA position, three galaxies are seen: $\sim 2^{\prime \prime}$ southeast, $\sim 0^{\prime \prime} 8$ southeast, and $\sim 1^{\prime \prime}$ southwest to the SMA position. We derive photometric redshifts for the sources and find that they are likely to be at $z<1$, and it is unlikely that they are related to the SMA source (see Section 5).

\section{SED FITTING}

We perform SED model fitting to the photometry data from UV to radio to estimate photometric redshifts and other physical properties (stellar mass, $V$-band extinction, star formation timescale, and metallicity). The photometric data are divided into two wavelength ranges: (1) from UV to $8.0 \mu \mathrm{m}$, where stellar emission is dominant, (2) from submillimeter to radio, where thermal dust and synchrotron emission are dominant.

\subsection{UV-Optical-IR}

SED fits are performed to the photometry ranging from UV to $8.0 \mu \mathrm{m}\left(u, B, V, R, i^{\prime}, z^{\prime}, J, H, K, 3.6 \mu \mathrm{m}, 4.5 \mu \mathrm{m}, 5.8 \mu \mathrm{m}\right.$, and $8.0 \mu \mathrm{m}$ ) using the Hyperz code (Bolzonella et al. 2000). We use a synthetic spectral library of GALAXEV (Bruzual \& Charlot 2003) for SED templates. We adopt the Calzetti 


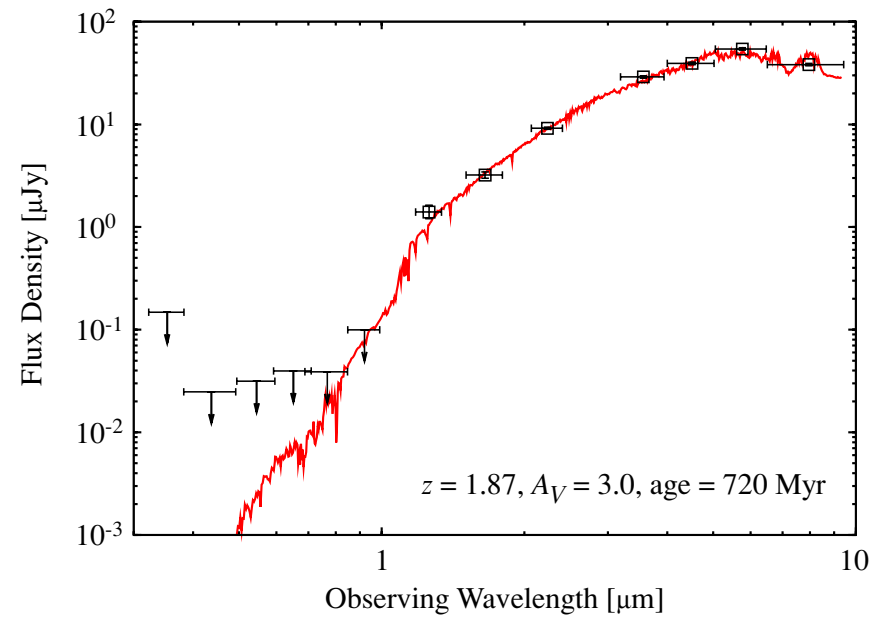

Figure 3. Best-fit SED obtained in UV-optical-IR SED fit along with photometry data. Downward arrows represent $3 \sigma$ upper limits.

Table 2

Best-fit Results in UV-Optical-IR SED Fit

\begin{tabular}{|c|c|c|c|c|c|c|}
\hline (1) & $\begin{array}{c}M_{\star} \\
\left(M_{\odot}\right) \\
(2)\end{array}$ & $\begin{array}{c}A_{V} \\
(\mathrm{mag}) \\
(3)\end{array}$ & $\begin{array}{c}\text { Age } \\
\text { (Myr) } \\
(4)\end{array}$ & $\begin{array}{c}\tau \\
(\mathrm{Myr}) \\
(5)\end{array}$ & $\begin{array}{c}Z \\
\left(Z_{\odot}\right) \\
(6)\end{array}$ & (7) \\
\hline $1.87_{-0.07}^{+0.15}$ & $2.5_{-0.3}^{+2.2} \times 10^{11}$ & $3.0_{-1.0}^{+0.3}$ & $720_{-210}^{+1880}$ & 20 & 1 & 13.4 \\
\hline
\end{tabular}

Notes. The errors are $68 \%$ confidence intervals. (1) Photometric redshift; (2) stellar mass; (3) $V$-band attenuation; (4) age; (5) star formation timescale; (6) metallicity; (7) $\chi^{2}$ value.

extinction law (Calzetti et al. 2000) and the Chabrier (2003) initial mass function with lower and upper cutoff masses of 0.1 and $100 M_{\odot}$. Two star formation histories are assumed: (1) exponentially decaying SFR with a timescale of $\tau$ (i.e., SFR $\left.\propto e^{-t / \tau}\right)$; and (2) constant SFR. We calculate $\chi^{2}$ values for each SED template with free parameters of redshift $(z=$ $0-6)$, stellar mass $\left(M_{\star}\right), V$-band attenuation $\left(A_{V}=0.0-6.0\right)$, star formation timescale ( $\tau=0.01-5 \mathrm{Gyr})$, and metallicity $\left(Z=1.0,0.4,0.2 Z_{\odot}\right)$. The best-fit results are obtained with the exponentially decaying SFR, $z=1.87_{-0.07}^{+0.15}, M_{\star}=$ $2.5_{-0.3}^{+2.2} \times 10^{11} M_{\odot}, A_{V}=3.0_{-1.0}^{+0.3} \mathrm{mag}$, and age $=720_{-210}^{+1880} \mathrm{Myr}$ (Table 2 and Figure 3). The results suggest that SXDF 850.6 has a large population of old stars, with large amount of dust obscuring the stellar emission. The stellar mass is comparable to those of massive star-forming galaxies at $z \sim 2$ (e.g., Shapley et al. 2005; Erb et al. 2006a) and consistent with stellar masses recently derived for other SMGs $\left(\sim 10^{11}-10^{12} M_{\odot}\right.$; e.g., Dye et al. 2008; Michałowski et al. 2009).

\subsection{Submillimeter-Radio}

Photometry at $850 \mu \mathrm{m}, 880 \mu \mathrm{m}, 1100 \mu \mathrm{m}$, and $21 \mathrm{~cm}$ is used for submillimeter-radio SED fits. The MIPS photometry is also used to check for consistency. We use the following starburst SED models: Arp 220 (Silva et al. 1998), the average SED of 76 SMGs with spectroscopic redshifts (Michałowski et al. 2009), and 105 SED models of Chary \& Elbaz (2001). We perform minimized $\chi^{2}$ fits with free parameters of redshifts and flux scaling factors. We find the best-fit redshifts of $z=2.1_{-0.4}^{+0.5}$ for Arp $220\left(\chi^{2}=0.3\right), z=1.8_{-0.3}^{+0.5}$ for the average SMG $\left(\chi^{2}=0.4\right)$, and $z=2.5_{-0.4}^{+0.6}$ for an SED of Chary \& Elbaz $\left(2001 ; \chi^{2}=0.09\right)$. The errors are $99 \%$ confidence intervals. Note that there are other templates in the library of Chary

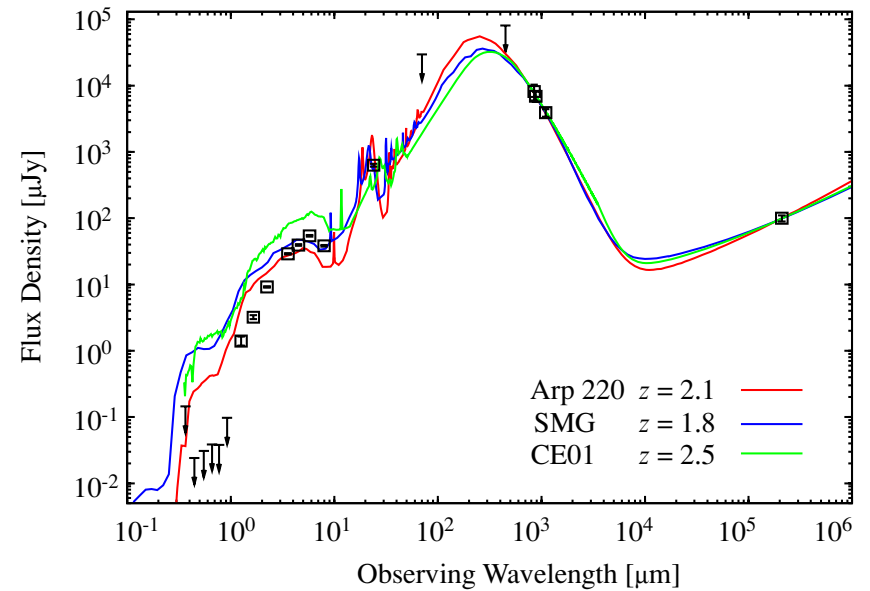

Figure 4. Best-fit SEDs for three different models obtained in submillimeterradio SED fit. The SEDs are the average SED of 76 SMGs (Michałowski et al. 2009) at $z=1.8$, Arp 220 (Silva et al. 1998) at $z=2.1$, and a starburst template of Chary \& Elbaz (2001) at $z=2.5$. Photometry data from UV to radio are overplotted.

\& Elbaz (2001) that fit the photometry data with similar $\chi^{2}$ values in the redshift range of $z \sim 1-3$. The derived redshifts are consistent with the result in the UV-optical-IR SED fit in the previous section. The IR luminosities are estimated to be $L_{\mathrm{IR}}=(7-26) \times 10^{12} L_{\odot}$ from the intrinsic IR luminosities of SED templates multiplied by scaling factors for the bestfit SEDs. The best-fit SED models are shown in Figure 4 with photometry data from UV to radio. All of the SEDs overestimate the UV-NIR photometry, suggesting that stellar emission is heavily attenuated by dust. This is consistent with the large extinction $\left(A_{V}=3.0\right)$ derived from the UV-optical-IR SED fit. Finally, we note that the SMG sample in Michałowski et al. (2009) is biased against optically faint objects. It is clearly important to take into account a heavily obscured SMG like SXDF 850.6 (or e.g., GOODS 850-5 in Wang et al. 2009; AzTEC1 in Y. Tamura et al. 2010, in preparation) to properly understand the overall picture of SMGs.

\section{DISCUSSION}

\subsection{AGN Contribution}

The rest-frame $1.6 \mu \mathrm{m}$ bump is clearly seen at $5.8 \mu \mathrm{m}$ (Figure 3), suggesting that the NIR emission detected in the IRAC bands is star formation dominated (e.g., Weedman et al. 2006; Farrah et al. 2008). The flatter spectral slope in the IRAC emission compared to AGN populations favors a star formation as a dominant heating source (Yun et al. 2008; Ivison et al. 2004). This is also supported by the fact that SXDF 850.6 appears close to the starburst model tracks in the $S_{8.0} / S_{4.5}$ versus $S_{24} / S_{8.0}$ color-color diagram of Ivison et al. (2004). These facts suggest that SXDF 850.6 favors star formation as a dominant heating source and the physical quantities derived in the SED fit are less affected by the AGN.

The $3 \sigma$ upper limit on the rest-frame X-ray luminosity derived from $0.2-12 \mathrm{keV}$ flux is $1.3 \times 10^{44} \mathrm{erg} \mathrm{s}^{-1}$ (assuming $z=1.87$ and an effective photon index of $\Gamma=1.8$ ). Since this value is not properly corrected for hydrogen attenuation, we do not constrain the AGN contribution from the X-ray luminosity.

\subsection{Dust Mass and Molecular Gas Mass}

Assuming the observed $880 \mu \mathrm{m}$ flux is dominated by thermal dust emission, the dust mass can be derived as 
$M_{d}=S_{\text {obs }} D_{L}^{2} /\left[(1+z) \kappa_{d}\left(v_{\text {rest }}\right) B\left(v_{\text {rest }}, T_{d}\right)\right]$ (e.g., Hughes et al. $1997)$, where $S_{\text {obs }}$ is the observed flux density, $v_{\text {rest }}$ is the restframe frequency, $\kappa_{d}\left(v_{\text {rest }}\right)$ is the dust mass absorption coefficient, $T_{d}$ is the dust temperature, and $B\left(v_{\text {rest }}, T_{d}\right)$ is the Planck blackbody function. We assume that the absorption coefficient varies as $\kappa_{d} \propto \nu^{\beta}$ and $\beta$ lies between 1 and 2 (e.g., Hildebrand 1983). We adopt $\kappa_{d}(125 \mu \mathrm{m})=2.64 \pm 0.29 \mathrm{~m}^{2} \mathrm{~kg}^{-1}$, the average value of various studies (Dunne et al. 2003), $T_{d}=30-50 \mathrm{~K}$, and $\beta=1.5$, the typical values for SMGs (e.g., Kovács et al. 2006; Pope et al. 2006; Coppin et al. 2008; Michałowski et al. 2009). The dust mass is estimated to be $M_{d}=(4-9) \times 10^{8} M_{\odot}$ for SXDF 850.6 at $z=1.87$. This is consistent with previous work on SMGs (e.g., Kovács et al. 2006; Coppin et al. 2008; Michałowski et al. 2009).

By adopting a gas-to-dust mass ratio of 54, which is an average value for SMGs in Kovács et al. (2006), the molecular gas mass is $M_{\text {gas }}=(2-5) \times 10^{10} M_{\odot}$.

\subsection{Star Formation Activity and Nature of SXDF 850.6}

The UV-IR SED exhibits quiescent star-forming activity dominated by old stellar components. The current SFR estimated from the SED fit is approximately zero. This is because the large part of the stellar mass was formed at the early phase of star formation and SFR decreased with time following exponential decay ( $\tau=20 \mathrm{Myr}$ for the best-fit SED). On the other hand, the IR luminosity of the best-fit submillimeter-radio SED provides $\mathrm{SFR}_{\mathrm{IR}}=1300-4500 M_{\odot} \mathrm{yr}^{-1}$ (Kennicutt 1998). The SFR derived from $1.4 \mathrm{GHz}$ radio emission is $1400 M_{\odot} \mathrm{yr}^{-1}$ following the equation of Bell (2003), and $1100 M_{\odot} \mathrm{yr}^{-1}$ following Yun \& Carilli (2002) (with a spectral index of -0.8 and a radio-FIR normalization factor of 1 ).

To see whether the observational data allow the coexistence of the old stellar SED with quiescent star formation and the dusty starburst SED, we create synthetic SEDs at UV-IR wavelength using the GALAXEV library. The synthetic SEDs are composites of an SED with the same parameters as the best-fit SED which is dominated by old stellar population and starburst SEDs with different parameters. We find that the composite SEDs with plausible parameters for starburst SEDs (e.g., SFR $2000 M_{\odot} \mathrm{yr}^{-1}, A_{V}=3.0$, and age = $1 \mathrm{Myr}$ ) are consistent with the photometry data (Figure 5). Note that combining the SEDs increases the total stellar mass by only $\sim 1 \%$.

Combining these results allows us to infer the nature of SXDF 850.6: it is a mature system with a large fraction of old stellar components, and currently experiencing a vigorous dusty starburst. The coexistence of old stars and a current starburst in an SMG is suggested by Wang et al. (2009) from a detailed SED analysis. SXDF 850.6 has enough molecular gas mass as estimated in Section 6.2 to maintain intense star formation. Such significant star formation is likely caused by major mergers (e.g., Greve et al. 2005; Tacconi et al. 2006, 2008; Narayanan et al. 2010). If the star formation continues with $S F R \sim$ a few $10^{3} M_{\odot} \mathrm{yr}^{-1}$, the gas consumption time is $\sim$ a few $10 \mathrm{Myr}$. This scenario is well described by hydrodynamic simulations of Narayanan et al. (2010) in which a major merger with a $\sim 10^{13} M_{\odot}$ dark matter halo produces a peak flux of $\sim 7-8 \mathrm{mJy}$ at $850 \mu \mathrm{m}$ for a short duration (<50 Myr). We are observing the short-lived glow of SXDF 850.6 as a bright SMG. Even if all the molecular gas converts into stars, the total stellar mass will increase by at most $30 \%$. This supports an idea that the large fraction of stellar mass in SMGs is constructed before the

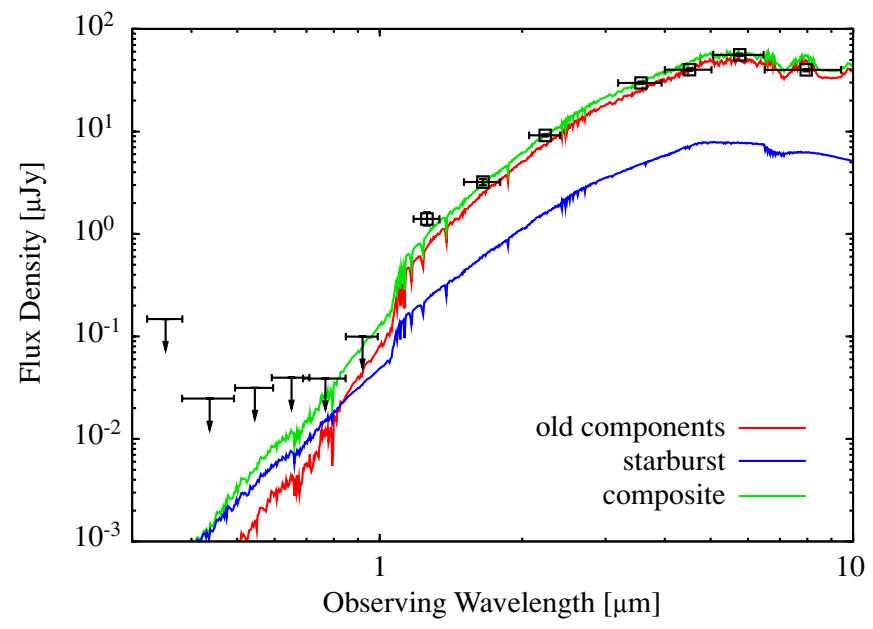

Figure 5. Synthetic SEDs of old stellar components (red), starburst components at the age of $100 \mathrm{Myr}$ with an initial SFR of $3000 M_{\odot} \mathrm{yr}^{-1}$ (blue), and a sum of both (green) created with the GALAXEV library. Photometry data are also shown.

current submillimeter bright phase (e.g., Borys et al. 2005; Dye et al. 2008; Michałowski et al. 2009).

The specific star formation rate (SSFR; SFR per unit stellar mass, an estimator of current star-forming activity) of $\sim 5$ $20 \mathrm{Gyr}^{-1}$ places SXDF 850.6 on the high mass end of the correlation between stellar mass and SFR for $z \sim 2$ star-forming galaxies or above the correlation (e.g., Erb et al. 2006b; Reddy et al. 2006; Daddi et al. 2007). If the large population of stars was constructed before the current starburst phase, the enhancement of star formation could be due to merging of massive starforming galaxies (e.g., merging BzKs; Takagi et al. 2008), and it is possible that this galaxy evolves into a massive present-day elliptical through major merger events (e.g., Lilly et al. 1999; Smail et al. 2004).

\section{SUMMARY}

We conducted SMA $880 \mu \mathrm{m}$ observations of a submillimeter galaxy, SXDF 850.6, detected with SCUBA in the Subaru/ $X M M-N e w t o n$ Deep Field, which has multiple radio and IR counterpart candidates. The precise astrometry with the SMA shows that the correct counterpart is the most distant radio source from the SCUBA coordinates. Although there is a lack of a corresponding source at the SMA position in deep images of $u$, $B, V, R, i^{\prime}, z^{\prime}$ bands, we find a counterpart at $J, H, K, 3.6 \mu \mathrm{m}$, $4.5 \mu \mathrm{m}, 5.8 \mu \mathrm{m}, 8.0 \mu \mathrm{m}, 24 \mu \mathrm{m}, 1100 \mu \mathrm{m}$, and $21 \mathrm{~cm}$. A detailed analysis of fitting a library of synthetic SEDs to the photometry from the $u$ band to $8.0 \mu \mathrm{m}$ gives the best-fit parameters of $z=1.87_{-0.07}^{+0.15}, M_{\star}=2.5_{-0.3}^{+2.2} \times 10^{11} M_{\odot}, A_{V}=$ $3.0_{-1.0}^{+0.3} \mathrm{mag}$, and age $=720_{-210}^{+1880} \mathrm{Myr}$. The SED fit to the submillimeter-radio photometry using the three different starburst SED models provides the consistent redshift of $z \sim 1.8$ 2.5 and $L_{\mathrm{IR}}=(7-26) \times 10^{12} L_{\odot}$. The IR color diagnostic suggests that the AGN contribution to the SED is small. The SFR derived from the IR luminosity is 1300-4500 $M_{\odot} \mathrm{yr}^{-1}$, and the SSFR is $\sim 10 \mathrm{Gyr}^{-1}$. If the intense starburst continues, the estimated molecular gas mass of $(2-5) \times 10^{10} M_{\odot}$ will last for a few Myr. Given these facts, it is suggested that SXDF 850.6 is a mature system that already contains a dominant fraction of its expected final stellar mass in an old stellar population, and which is also experiencing a significant starburst. The enhancement of star formation could be due to major mergers. 
We thank the anonymous referee for valuable comments. B.H. is financially supported by a Research Fellowship from the JSPS for Young Scientists. J.S.D. acknowledges the support of the Royal Society through a Wolfson Research Merit award.

\section{REFERENCES}

Alexander, D. M., Bauer, F. E., Chapman, S. C., Smail, I., Blain, A. W., Brandt, W. N., \& Ivison, R. J. 2005, ApJ, 632, 736

Austermann, J. E., et al. 2009, MNRAS, 393, 1573

Barger, A. J., Cowie, L. L., \& Richards, E. A. 2000, AJ, 119, 2092

Barger, A. J., Cowie, L. L., Sanders, D. B., Fulton, E., Taniguchi, Y., Sato, Y., Kawara, K., \& Okuda, H. 1998, Nature, 394, 248

Bell, E. F. 2003, ApJ, 586, 794

Bertin, E., \& Arnouts, S. 1996, A\&AS, 117, 393

Blain, A. W., Smail, I., Ivison, R. J., Kneib, J.-P., \& Frayer, D. T. 2002, Phys. Rep., 369, 111

Bolzonella, M., Miralles, J. M., \& Pelló, R. 2000, A\&A, 363, 476

Borys, C., Smail, I., Chapman, S. C., Blain, A. W., Alexander, D. M., \& Ivison, R. J. 2005, ApJ, 635, 853

Bruzual, G., \& Charlot, S. 2003, MNRAS, 344, 1000

Calzetti, D., Armus, L., Bohlin, R. C., Kinney, A. L., Koornneef, J., \& StorchiBergmann, T. 2000, ApJ, 533, 682

Capak, P., et al. 2008, ApJ, 681, L53

Chabrier, G. 2003, ApJ, 586, L133

Chapman, S. C., Blain, A. W., Smail, I., \& Ivison, R. J. 2005, ApJ, 622, 772

Chary, R., \& Elbaz, D. 2001, ApJ, 556, 562

Clements, D. L., et al. 2008, MNRAS, 387, 247

Coppin, K., et al. 2006, MNRAS, 372, 1621

Coppin, K., et al. 2008, MNRAS, 384, 1597

Coppin, K., et al. 2009, MNRAS, 395, 1905

Daddi, E., et al. 2007, ApJ, 670, 156

Daddi, E., et al. 2009, ApJ, 694, 1517

Downes, A. J. B., Peacock, J. A., Savage, A., \& Carrie, D. R. 1986, MNRAS, 218,31

Dunne, L., Eales, S. A., \& Edmunds, M. G. 2003, MNRAS, 341, 589

Dye, S., et al. 2008, MNRAS, 386, 1107

Erb, D. K., Steidel, C. C., Shapley, A. E., Pettini, M., Reddy, N. A., \& Adelberger, K. L. 2006a, ApJ, 646, 107

Erb, D. K., Steidel, C. C., Shapley, A. E., Pettini, M., Reddy, N. A., \& Adelberger, K. L. 2006b, ApJ, 647, 128

Ezawa, H., Kawabe, R., Kohno, K., \& Yamamoto, S. 2004, Proc. SPIE, 5489, 763

Ezawa, H., et al. 2008, Proc. SPIE, 7012, 6

Farrah, D., et al. 2008, ApJ, 677, 957

Furusawa, H., et al. 2008, ApJS, 176, 1

Greve, T. R., Ivison, R. J., Bertoldi, F., Stevens, J. A., Dunlop, J. S., Lutz, D., \& Carilli, C. L. 2004, MNRAS, 354, 779

Greve, T. R., et al. 2005, MNRAS, 359, 1165

Hildebrand, R. H. 1983, Q. J. R. Astron. Soc., 24, 267

Ho, P. T. P., Moran, J. M., \& Lo, K. Y. 2004, ApJ, 616, L1

Hughes, D. H., Dunlop, J. S., \& Rawlings, S. 1997, MNRAS, 289, 766

Hughes, D. H., et al. 1998, Nature, 394, 241

Iono, D., et al. 2006a, ApJ, 640, 1
Iono, D., et al. 2006b, ApJ, 645, 97

Ivison, R. J., Smail, I., Barger, A. J., Kneib, J. P., Blain, A. W., Owen, F. N., Kerr, T. H., \& Cowie, L. L. 2000, MNRAS, 315, 209

Ivison, R. J., Smail, I., Le Borgne, J.-F., Blain, A. W., Kneib, J.-P., Bezecourt, J., Kerr, T. H., \& Davies, J. K. 1998, MNRAS, 298, 583

Ivison, R. J., et al. 2002, MNRAS, 337, 1

Ivison, R. J., et al. 2004, ApJS, 154, 124

Ivison, R. J., et al. 2005, MNRAS, 364, 1025

Ivison, R. J., et al. 2007, MNRAS, 380, 199

Ivison, R. J., et al. 2008, MNRAS, 390, 1117

Kennicutt, R. C., Jr. 1998, ARA\&A, 36, 189

Kovács, A., Chapman, S. C., Dowell, C. D., Blain, A. W., Ivison, R. J., Smail, I., \& Phillips, T. G. 2006, ApJ, 650, 592

Lacy, M., et al. 2005, ApJS, 161, 41

Laurent, G. T., et al. 2005, ApJ, 623, 742

Lilly, S. J., Eales, S. A., Gear, W. K. P., Hammer, F., Le Fèvre, O., Crampton, D., Bond, J. R., \& Dunne, L. 1999, ApJ, 518, 641

Michałowski, M. J., Hjorth, J., \& Watson, D. 2009, arXiv:0905.4499

Mortier, A. M., et al. 2005, MNRAS, 363, 563

Narayanan, D., Hayward, C. C., Cox, T. J., Hernquist, L., Jonsson, P., Younger, J. D., \& Groves, B. 2010, MNRAS, 401, 1613

Perera, T. A., et al. 2008, MNRAS, 391, 1227

Pope, A., et al. 2006, MNRAS, 370, 1185

Reddy, N. A., Steidel, C. C., Fadda, D., Yan, L., Pettini, M., Shapley, A. E., Erb, D. K., \& Adelberger, K. L. 2006, ApJ, 644, 792

Sault, R. J., Teuben, P. J., \& Wright, M. C. H. 1995, ASP Conf. Ser. 77, Astronomical Data Analysis Software and Systems IV (San Francisco, CA: ASP), 433

Scott, et al. 2002, MNRAS, 331, 817

Scott, K. S., et al. 2008, MNRAS, 385, 2225

Scoville, N. Z., Carlstrom, J. E., Chandler, C. J., Phillips, J. A., Scott, S. L., Tilanus, R. P. J., \& Wang, Z. 1993, PASP, 105, 1482

Shapley, A. E., Steidel, C. C., Erb, D. K., Reddy, N. A., Adelberger, K. L., Pettini, M., Barmby, P., \& Huang, J. 2005, ApJ, 626, 698

Silva, L., Granato, G. L., Bressan, A., \& Danese, L. 1998, ApJ, 509, 103

Smail, I., Chapman, S. C., Blain, A. W., \& Ivison, R. J. 2004, ApJ, 616, 71

Smail, I., Ivison, R. J., \& Blain, A. W. 1997, ApJ, 490, 5

Smail, I., Ivison, R. J., Owen, F. N., Blain, A. W., \& Kneib, J.-P. 2000, ApJ, 528,612

Tacconi, L. J., et al. 2006, ApJ, 640, 228

Tacconi, L. J., et al. 2008, ApJ, 680, 246

Takagi, T., Ono, Y., Shimasaku, K., \& Hanami, H. 2008, MNRAS, 389, 775

Tamura, Y., et al. 2009, Nature, 459, 61

Wagg, J., Owen, F., Bertoldi, F., Sawitzki, M., Carilli, C. L., Menten, K. M., \& Voss, H. 2009, ApJ, 699, 1843

Wang, W.-H., Barger, A. J., \& Cowie, L. L. 2009, ApJ, 690, 319

Wang, W.-H., Cowie, L. L., van Saders, J., Barger, A. J., \& Williams, J. P. 2007, ApJ, 670, L89

Weedman, D., et al. 2006, ApJ, 653, 101

Wilson, G. W., et al. 2008, MNRAS, 386, 807

Younger, J. D., et al. 2007, ApJ, 671, 1531

Younger, J. D., et al. 2008, MNRAS, 387, 707

Younger, J. D., et al. 2009, ApJ, 704, 803

Yun, M. S., \& Carilli, C. L. 2002, ApJ, 568, 88

Yun, M. S., et al. 2008, MNRAS, 389, 333 
ERRATUM: “UNVEILING THE NATURE OF SUBMILLIMETER GALAXY SXDF 850.6” (2010, ApJ, 711, 974)

B. Hatsukade ${ }^{1}$, D. Iono ${ }^{2}$, T. Yoshikawa ${ }^{3}$, M. AkiYAma $^{3}$, J. S. Dunlop ${ }^{4}$, R. J. Ivison ${ }^{4,5}$, A. B. PeCK ${ }^{6}$, S. IKarashi $^{1}$, A. BigGs $^{7}$, H. Ezawa ${ }^{8}$, H. Hanami ${ }^{9}$, P. Ho ${ }^{10}$, D. H. Hughes ${ }^{11}$, R. Kawabe ${ }^{2}$, K. Kohno $^{1,12}$, S. Matsushita ${ }^{10}$, K. NAKANishi ${ }^{2}$,

N. Padilla ${ }^{13}$, G. PetitPas ${ }^{14}$, Y. TAmura ${ }^{2}$, J. WagG ${ }^{11,15}, 16$, D. J. Wilner ${ }^{16}$, G. W. Wilson ${ }^{17}$, T. YAMAdA $^{3}$, And M. S. Yun ${ }^{17}$

${ }^{1}$ Institute of Astronomy, University of Tokyo, 2-21-1 Osawa, Mitaka, Tokyo 181-0015, Japan; hatsukade@ioa.s.u-tokyo.ac.jp

${ }^{2}$ Nobeyama Radio Observatory, Minamimaki, Minamisaku, Nagano 384-1805, Japan

${ }^{3}$ Astronomical Institute, Tohoku University, Aramaki, Aoba-ku, Sendai, Miyagi 980-8578, Japan

${ }^{4}$ Scottish Universities Physics Alliance, Institute for Astronomy, School of Physics and Astronomy, University of Edinburgh, Royal Observatory, Edinburgh EH9 3HJ, UK

${ }^{5}$ UK Astronomy Technology Centre, Science and Technology Research Council, Royal Observatory, Blackford Hill, Edinburgh EH9 3HJ, UK

6 Joint ALMA Observatory, Avenida El Golf 40, Piso 18, Las Condes 7550108 Santiago, Chile

${ }^{7}$ European Southern Observatory, Karl-Schwarzschild-Straße 2, D-85748 Garching, Germany

${ }^{8}$ National Astronomical Observatory of Japan, 2-21-1 Osawa, Mitaka, Tokyo 181-8588, Japan

${ }^{9}$ Physics Section, Faculty of Humanities and Social Sciences, Iwate University, Morioka 020-8550, Japan

10 Academia Sinica Institute of Astronomy and Astrophysics, P.O. Box 23-141, Taipei 10617, Taiwan

${ }^{11}$ Instituto Nacional de Astrofisica, Óptica y Electrónica (INAOE), Apartado Postal 51 y 216, 72000 Puebla, Pue., Mexico

${ }^{12}$ Research Center for the Early Universe, University of Tokyo, 7-3-1 Hongo, Bunkyo, Tokyo 113-0033, Japan

${ }^{13}$ Departamento de Astronomía y Astrofísica, Pontificia Universidad Católica de Chile, Vicuña Mackenna 4860, Santiago, Chile

${ }^{14}$ Harvard-Smithsonian Center for Astrophysics, Submillimeter Array, 645 North A'ohoku Place, Hilo, HI 96720, USA

${ }^{15}$ European Southern Observatory, Alonso de Córdova 3107, Vitacura, Casilla 19001, Santiago 19, Chile

${ }^{16}$ Harvard-Smithsonian Center for Astrophysics, 60 Garden Street, Cambridge, MA 02138, USA

${ }^{17}$ Department of Astronomy, University of Massachusetts, Amherst, MA 01003, USA

Due to an error at the publisher, the names of the third and fourth authors of this paper were incorrectly printed. We print here the correct names of the authors. IOP Publishing sincerely regrets this error. 